\title{
Influence of sclerotomy use on vitreous incarceration in an experimental model of vitrectomized eye
}

This article was published in the following Dove Press journal:

Clinical Ophthalmology

18 July 2013

Number of times this article has been viewed

Javier Benitez-Herreros'

Lorenzo Lopez-Guajardo 1,2

Cristina Camara-Gonzalez'

Aurora Perez-Crespo'

Agustin Silva-Mato ${ }^{3}$

Miguel A Teus ${ }^{1,2}$

'Department of Ophthalmology, University Hospital Príncipe de Asturias, Madrid, Spain; ${ }^{2}$ Department of Surgery, Medical and Social Sciences, Faculty of Medicine, Alcalá University, Madrid, Spain; ${ }^{3}$ Department of Health and Sociomedical Sciences, Alcalá University, Madrid, Spain
Correspondence: Lorenzo LopezGuajardo

Department of Ophthalmology, University Hospital Príncipe de Asturias, Carretera Alcalá-Meco, Alcalá de Henares, Madrid 28805, Spain

Email Ilguajardo@icam.es
Purpose: To evaluate the influence of sclerotomy use during vitrectomy (vitreous cutter, illumination probe, or infusion-line entrance) on postoperative vitreous incarceration using an experimental model of vitrectomized eye.

Materials and methods: Experimental, randomized, and observer-masked study in which 23-gauge transconjunctival sutureless vitrectomy was performed in cadaveric pig eyes. Postoperative incisional vitreous entrapment was evaluated by direct visualization. No vitreous incarceration was classified as grade $0(\mathrm{G} 0)$, thin vitreous entrapment was classified as grade 1 (G1), and thick vitreous strands as grade 2 (G2).

Results: A total of 46 eyes were included. Vitreous incarceration was detected in $91.3 \%$ $(43.5 \% \mathrm{G} 1,47.8 \% \mathrm{G} 2)$ of the sclerotomies used by the vitreous cutter probe, $95.7 \%$ (45.7\% G1, 50\% G2) of the illumination-pipe entrances, and $93.5 \%(45.7 \% \mathrm{G} 1,47.8 \% \mathrm{G} 2)$ of the infusion-line incisions. No statistical differences were found when comparing incisional vitreous incarceration after vitrectomy according to sclerotomy use.

Conclusion: Different manipulation of the sclerotomies, depending on their use, does not seem to influence postvitrectomy vitreous entrapment in our experimental model.

Keywords: vitrectomy, vitreous incarceration, direct visualization, sclerotomy use, experimental model

\section{Introduction}

Pars plana vitrectomy was first developed by Machemer et al in $1972 .{ }^{1}$ Many advances have since been developed in vitrectomy techniques and instrumentation. Since the implementation of transconjunctival sutureless vitrectomy (TSV), ${ }^{2,3}$ numerous studies have analyzed different aspects of sclerotomies, such as the presence of vitreous incarceration in sclerotomies. ${ }^{4-11}$ This vitreous entrapment may work as an incisional plug, favoring postoperative sclerotomy closure; nevertheless, it has also been related to some postoperative complications, such as peripheral retinal tears, ${ }^{12-14}$ acute endophthalmitis, ${ }^{15}$ and fibrovascular proliferation. ${ }^{16}$ Vitreous shaving around the sclerotomy sites ${ }^{17}$ and removing the scleral cannulas over the light probe at the end of the vitrectomy ${ }^{18}$ have been related to less postoperative vitreous incarceration.

When performing vitrectomy, the vitreous cutter (usually dominant hand) ordinarily rotates more around the sclerotomy and reaches more extreme positions inside the eye, when compared to the illumination probe (usually nondominant hand). This increase in manipulation would induce a wider dilation of the sclerotomy, and secondarily would influence the postoperative vitreous incarceration rate. In our study, we analyze the effect of sclerotomy use on incisional vitreous entrapment immediately after TSV, 
by direct visualization. This evaluation method is superior to other indirect viewing techniques, such as ultrasound biomicroscopy (UBM) or anterior-segment optical coherence tomography (AS-OCT), in the detection of vitreous entrapment. ${ }^{19}$ With the aim of analyzing in detail the influence that sclerotomy use may exert on postoperative vitreous incarceration, we considered it appropriate to study not only the rate but also the amount of the vitreous incarceration observed in the scleral incisions. Given the invasive nature of the procedure performed, we resolved to use an experimental animal model. The fact that pig eyes are easy to obtain, as well as their similarity with human globes, ${ }^{20-23}$ justified the choice of porcine eyeballs. Previously, in another animal sample, we analyzed the effect of the cannula-extraction technique on vitreous incarceration. ${ }^{18}$

\section{Materials and methods}

This experimental, randomized, observer-masked study included 46 pig eyes (Sus scrofa domesticus species). Exclusion criteria were presence of refractive media opacities, scleral alterations, and intraoperative complications. All eyes were obtained 3 hours after pig death and were kept in cold storage at $4^{\circ} \mathrm{C}$ before use, with the aim of preserving the properties of all ocular tissues, including the vitreous gel. ${ }^{24}$ Given that the globes were obtained from pigs killed for human consumption from a regulated slaughterhouse, no statements for the use of animals in ophthalmic research were applicable.

The 23-gauge sclerotomies (23-gauge TSV system; Alcon Laboratories, Fort Worth, TX, USA) were performed using an oblique incisional technique $4 \mathrm{~mm}$ from the limbus. ${ }^{25}$ One of the authors (LLG) performed vitrectomy with the infusion pressure set at $40 \mathrm{mmHg}$, until the vitreous cavity was judged optically empty, using the Accurus vitrectomy system (Alcon Laboratories). Vitrectomy was also performed all around each perisclerotomy area by inserting the vitrectomy probe through both superior cannulas. After checking that balanced salt solution flowed freely through the superior cannulas, the infusion pressure was lowered to $5 \mathrm{mmHg}$ and the superior cannulas were extracted with the plug inserted, following their oblique incisional pathways. Finally, intraocular pressure was raised to $10 \mathrm{mmHg}$, the infusion line was clamped, and the infusion cannula was taken out with the clamped infusion line inserted.

Eyes were cut and divided in two portions through the posterior third coronal plane of the globe. We first performed a penetrating incision parallel to the limbus with a scalpel, and then we completed the cut around the globe with Westcott scissors. Finally, slit-lamp photographs of the internal sides of the sclerotomies were taken (Figure 1). The image sequences obtained were reviewed, and one snapshot from each sclerotomy was selected for presentation in a masked fashion to one of the authors (JBH), who classified the sclerotomies according to the grade of vitreous incarceration in the wound. Grade 0 (G0) was defined as absence of vitreous incarceration at the sclerotomy site, grade 1 (G1) was considered as the presence of delicate vitreous strands directed to the inner hole of the incision, and grade 2 (G2) was classified as the existence of thick vitreous strands aimed toward the sclerotomy (Figure 2). The injection of triamcinolone acetonide, which stained the intraocular residual vitreous, allowed us to visualize the incisional vitreous strands clearly.

\section{Statistics}

For all statistical tests, $P<0.05$ was considered the significance level. To compare the effect of sclerotomy use (vitrectomy, light pipe, or infusion line) on the presence or absence

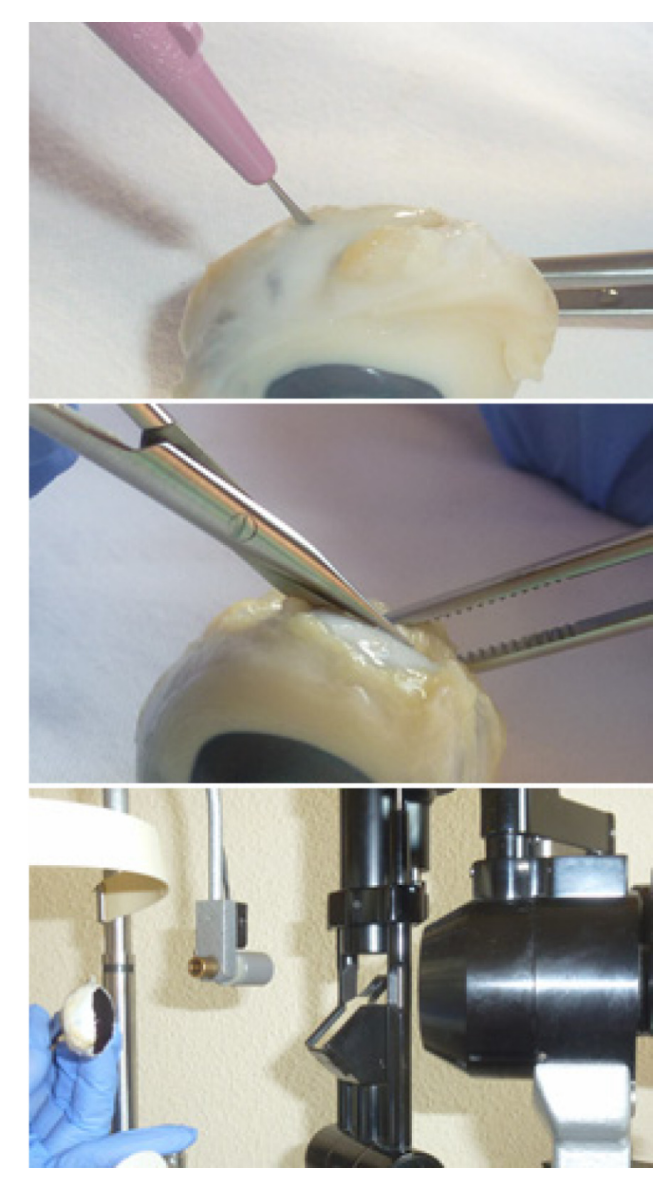

Figure I Eyes were cut and divided in two portions. We first performed a penetrating incision parallel to the limbus with a scalpel (top); then, we completed the cut around the globe with Westcott scissors (center); finally, slit-lamp photographs of the internal sides of the sclerotomies were taken (bottom). 

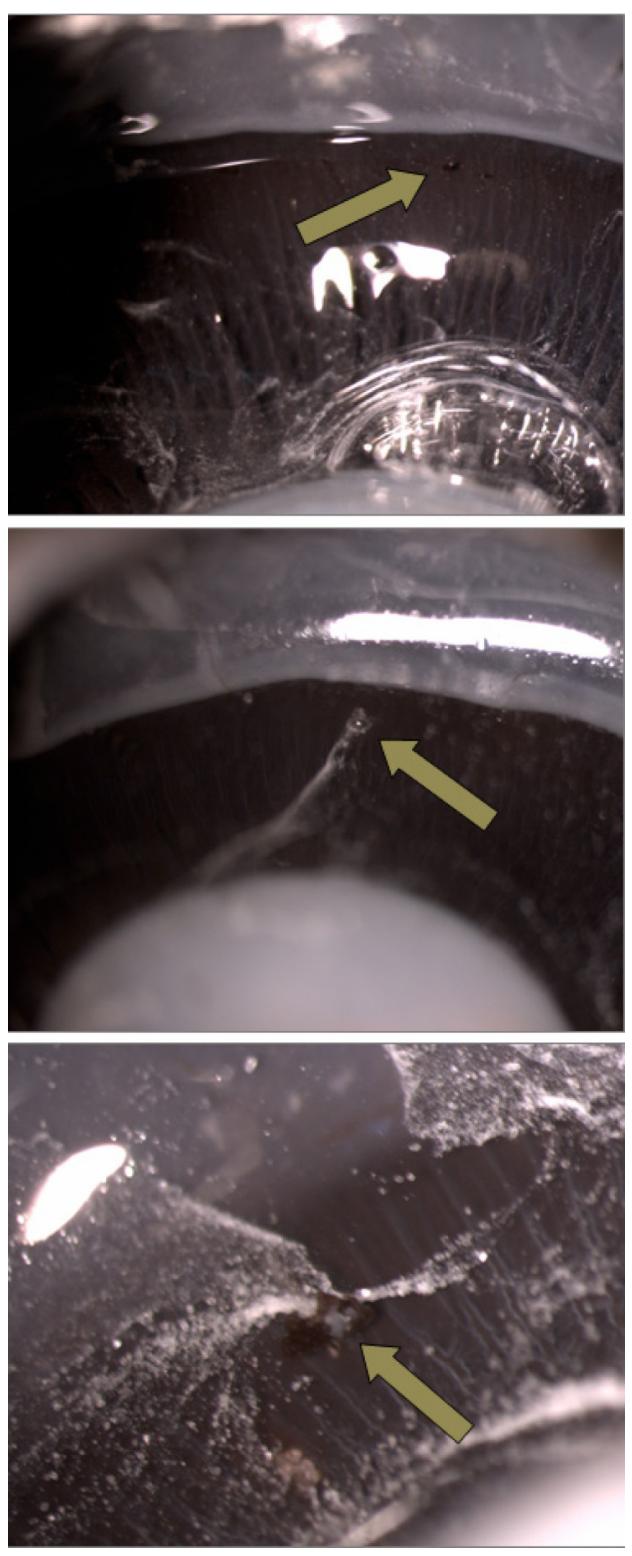

Figure 2 Sclerotomy slit-lamp photographs. The top photo was identified as absence of vitreous entrapment (grade 0), the center photo was classified as presence of delicate vitreous strands directed to the inner hole of the incision (grade I), and the bottom photo was classified as existence of thick vitreous strands aimed toward the sclerotomy (grade 2).

of vitreous incarceration, without considering the amount of incisional vitreous in grades ( $\mathrm{G} 0$ versus $\mathrm{G} 1+\mathrm{G} 2)$, we used the Chi-squared test. In turn, to compare the vitreous entrapment grades (G0, G1, or G2) obtained in the different sclerotomy groups, we used the Kruskal-Wallis test. Finally, to analyze the vitreous incarceration rate and grade found in superior sclerotomies (manipulated by dominant or nondominant hand) compared to infusion sclerotomies, used as controls, we employed the chi-squared test and the Mann-Whitney $U$ test, respectively. Statistical analyses were performed using StatView + Graphics (SAS Institute, Cary, NC, USA).

\section{Results}

Postmortem vitreous was clear in all globes. Most eyes did not present posterior vitreous detachment; however, for studying vitreous incarceration in anterior sclerotomies, we did not need to extract posterior hyaloid but just enough core and anterior vitreous to allow balanced salt solution to flow freely through the cannulas after removing the instruments. No intraoperative complications, such as retinal breaks or retinal detachments, were detected in any case.

Considering the 46 sclerotomies used by the dominant hand (vitrectomy probe), four had no signs of vitreous entrapment (G0 8.7\%), 20 had minimal vitreous strands incarcerated (G1 43.5\%), and 22 had thick vitreous strands entrapped (G2 47.8\%). Taking into account the sclerotomies used by the nondominant hand (illumination pipe), two had no signs of vitreous incarceration (G0 4.3\%), 21 presented minimal vitreous entrapment (G1 45.7\%), and 23 showed intense vitreous incarceration (G2 50\%). Regarding the incisions used by the infusion line, three were free of vitreous entrapment (G0 6.5\%), 21 had delicate vitreous strands (G1 45.7\%), and 22 presented incarcerated thick vitreous strands (G2 47.8\%) (Figure 3).

When the presence of incisional vitreous incarceration was analyzed in the sclerotomies according to their use, without considering the amount of vitreous entrapment in grades (G0 versus $\mathrm{G} 1+\mathrm{G} 2$ ), we did not find significant differences ( $P=0.7$, chi-squared test). In turn, when we compared the vitreous incarceration grades $(\mathrm{G} 0, \mathrm{G} 1$, or G2) observed in each sclerotomy group, we did not find significant differences either $(P=0.92$, Kruskal-Wallis test). Finally, when the rate and the grades of vitreous incarceration observed in superior sclerotomies (vitreous cutter and light-probe sclerotomies) were compared to vitreous entrapment in infusion sclerotomies, used as controls, no significant differences were found ( $P=1$, Chi-squared test; $P=0.91$, Mann-Whitney $U$ test).

\section{Discussion}

The risk of postoperative complications due to the presence of vitreous incarceration ${ }^{12-16}$ has encouraged investigators to analyze the influence that sclerotomy use may have on vitreous entrapment rate after vitrectomy. In that sense, Bhende et $\mathrm{al}^{26}$ and Yeh et $\mathrm{al}^{27}$ evaluated the presence of incisional vitreous after 20 -gauge vitrectomy. These studies, performed with UBM 2 months after the operations due to the risk of microbial contamination of the fresh wounds, revealed no significant differences in vitreous incarceration rates between the three incision groups compared. 


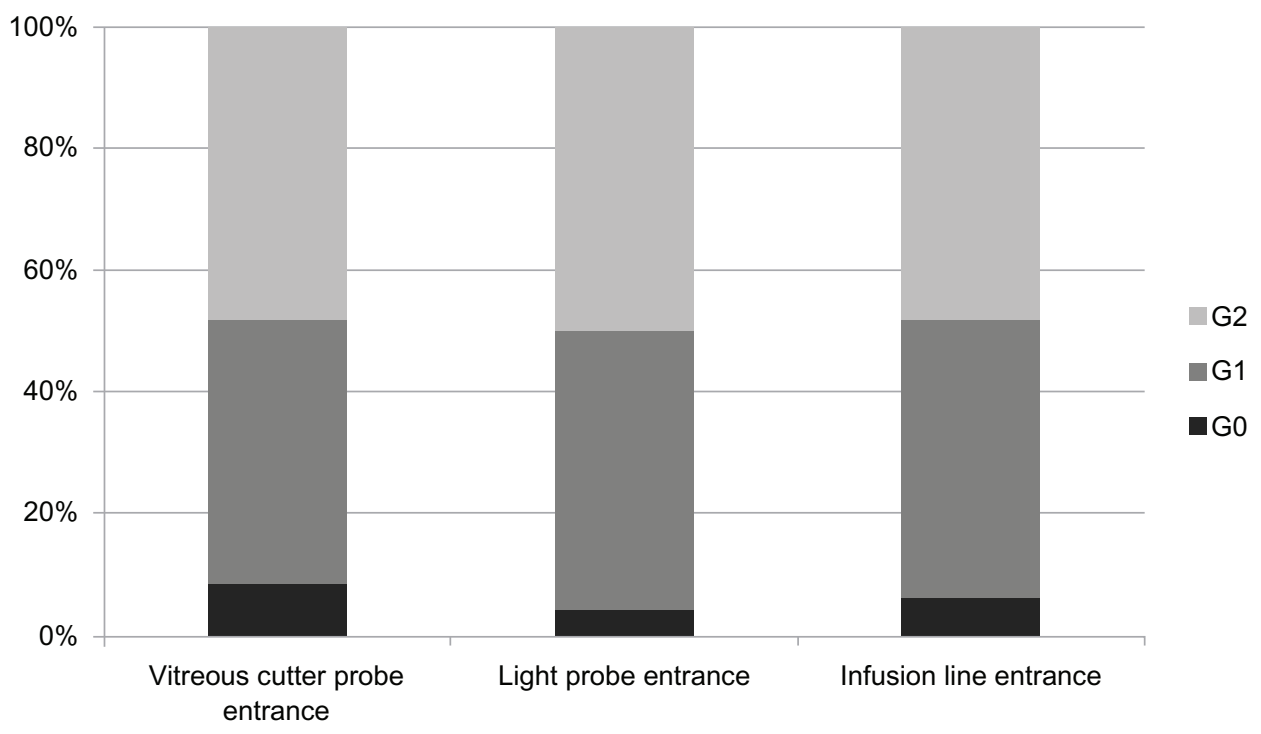

Figure 3 Proportion of vitreous incarceration grades (G0, GI, G2) in sclerotomies, according to the sclerotomy used (vitreous cutter-probe entrance, light-probe entrance, or infusion-line entrance entrance).

The establishment of 23- and 25-gauge TSV ${ }^{2,3}$ as firstchoice vitrectomy techniques for most vitreoretinal surgeons, and the differences they possess compared to conventional 20 -gauge vitrectomy (oblique sclerotomies, narrower gauge, and no suture at the end of the surgery), make it necessary to deeply reassess the influence of sclerotomy use on postoperative vitreous incarceration in these microincisional sclerotomies. Ahmadabadi et $\mathrm{al}^{9}$ evaluated with UBM the presence of vitreous incarceration in 30 eyes operated on with 20-, 23-, or 25-gauge incisions 8 weeks prior, without finding differences between the vitreous entrapment rates obtained in the sclerotomies determined by their use. However, it may be also interesting to analyze, closer to the vitrectomy end, the effect that the incision use may have not only on the rate but also on the amount (grade) of the sclerotomy vitreous entrapment. We resolved to use direct visualization as the evaluation method, given that it is more sensitive than indirect viewing techniques, such as UBM and AS-OCT, for identifying vitreous incarceration. ${ }^{19}$

In our study, we did not find differences in the postoperative vitreous incarceration rates $(\mathrm{G} 0$ vs $\mathrm{G} 1+\mathrm{G} 2)$ obtained in the sclerotomies despite their different uses (dominant-hand probe, nondominant-hand probe, or infusion-line entrance); these results agree with those obtained previously., ${ }^{9,26,27}$ In turn, we did not find differences in incisional vitreous incarceration grades after vitrectomy (G0, G1, or G2). Therefore, extreme maneuvers performed by the vitreous cutter probe, which would induce a wider dilation of the sclerotomy, do not seem to influence the rate or amount of immediate postoperative vitreous entrapment. Previous studies have not found any effect of incision use on other aspects of sclerotomies after vitrectomy, such as mechanical resistance of wounds ${ }^{28}$ or scleral healing. ${ }^{26}$

Despite the morphological similarities between human and pig eyes in scleral thickness ${ }^{20-22}$ and vitreous composition, ${ }^{23}$ absolute rates and grades of postoperative vitreous entrapment in sclerotomies obtained with our model should not be considered. The important outcome of our study is the lack of difference in postoperative vitreous incarceration obtained between the incisions compared. It would also be interesting to analyze the influence of sclerotomy use on vitreous incarceration in human eyes.

The use of this animal model allowed us to evaluate the sclerotomies in the immediate postoperative period, unlike human studies, in which UBM was performed around 2 months after surgery., ${ }^{9,26}$ We considered it useful to examine the sclerotomies shortly after the vitrectomy was finished, given that the vitreous incarcerated in the first days after the operation is related to postvitrectomy endophthalmitis. ${ }^{15}$ The time gap between vitrectomy and UBM exploration may alter the presence of the early postvitrectomy incisional vitreous, making results less reliable.

In view of our results, different uses of sutureless oblique sclerotomies (vitreous cutter, illumination-probe, or infusion-line entrance) in our animal model do not seem to influence incisional vitreous incarceration after vitrectomy, and therefore it may not be necessary to take any special care during or after surgery with any of the sclerotomies according to their use. If these results were confirmed in human eyes, it may not be necessary to perform a more 
intense vitrectomy around any of the incision sites based on use.

Our animal model may become the basis for future studies to analyze the influence that other factors may exert on incisional vitreous incarceration after vitrectomy, such as the presence of incisional leakage after cannula removal, the performance of additional surgical maneuvers (resection of retinal vasoproliferative tissue or macular membrane peeling), the length and angle of the scleral tunnels, the shape of the sclerotomies (linear or V-shaped incisions), the presence of residual vitreous inside the eye after the vitrectomy, and the intraocular pressure value during cannula extraction. In spite of our visualization method being more sensitive than other indirect-viewing techniques (UBM and AS-OCT) in the detection of vitreous incarceration, ${ }^{19}$ cutting the eyes could raise the intraocular pressure, increasing the amount of vitreous incarceration and making results less reliable. Therefore, in future studies, it would be interesting to analyze the presence of vitreous entrapment by another direct-viewing method, such as inserting a 23-gauge endoscopic video probe through any of the sclerotomies previously created; this technique would not increase intraocular pressure.

\section{Disclosure}

The authors report no conflicts of interest in this work.

\section{References}

1. Machemer R, Parel JM, Norton EW. Vitrectomy: a pars plana approach. Technical improvements and further results. Trans Am Acad Ophthalmol Otolaryngol. 1972;76(2):462-466.

2. Fujii GY, De Juan E Jr, Humayun MS, et al. A new 25-gauge instrument system for transconjunctival sutureless vitrectomy surgery. Ophthalmology. 2002;109(10):1807-1812.

3. Eckardt C. Transconjunctival sutureless 23-gauge vitrectomy. Retina. 2005;25(2):208-211.

4. Chen D, Lian Y, Cui L, Lu F, Ke Z, Song Z. Sutureless vitrectomy incision architecture in the immediate postoperative period evaluated in vivo using optical coherence tomography. Ophthalmology. 2010;117(10): 2003-2009.

5. Lopez-Guajardo L, Vleming-Pinilla E, Pareja-Esteban J, Teus-Guezala MA. Ultrasound biomicroscopy study of direct and oblique 25-gauge vitrectomy sclerotomies. Am J Ophthalmol. 2007;143(5):881-883.

6. Zhengyu S, Fang W, Ying F, Qinghua Q. The experimental research of rabbit's sclerotomy sites undergoing transconjunctival sutureless vitrectomy. Curr Eye Res. 2007;32(7-8):647-652.

7. Gutfleisch M, Dietzel M, Heimes B, Spital G, Pauleikhoff D, Lommatzsch A. Ultrasound biomicroscopic findings of conventional and sutureless sclerotomy sites after 20-, 23-, and 25-G pars plana vitrectomy. Eye (Lond). 2010;24(7):1268-1272.

8. Avitabile T, Castiglione F, Bonfiglio V, Castiglione F. Transconjunctival sutureless 25-gauge versus 20-gauge standard vitrectomy: correlation between corneal topography and ultrasound biomicroscopy measurements of sclerotomy sites. Cornea. 2010;29(1):19-25.
9. Ahmadabadi MN, Azaripour E, Movassat M, et al. Ultrasound biomicroscopy of conventional and sutureless $(23,25$-gauge) sclerotomy sites after pars plana vitrectomy. Iran J Ophthalmol. 2010;22(3):17-22.

10. Nagpal M, Wartikar S, Nagpal K. Comparison of clinical outcomes and wound dynamics of sclerotomy ports of 20, 25, and 23 gauge vitrectomy. Retina. 2009;29(2):225-231.

11. Inoue M, Ota I, Taniuchi S, Nagamoto T, Miyake K, Hirakata A. Miyake-Apple view of inner side of sclerotomy during microincision vitrectomy surgery. Acta Ophthalmol. 2011;89(5):412-416.

12. Buettner H, Machemer R. Histopathologic findings in human eyes after pars plana vitrectomy and lensectomy. Arch Ophthalmol. 1977;95(11): 2029-2033.

13. Gosse E, Newsom R, Lochhead J. The incidence and distribution of iatrogenic retinal tears in 20-gauge and 23-gauge vitrectomy. Eye (Lond). 2012;26(1):140-143.

14. Tan HS, Mura M, de Smet MD. Iatrogenic retinal breaks in 25-gauge macular surgery. Am J Ophthalmol. 2009;148(3):427-430.

15. Chen SD, Mohammed Q, Bowling B, Patel CK. Vitreous wick syndrome - a potential cause of endophthalmitis after intravitreal injection of triamcinolone through the pars plana. Am J Ophthalmol. 2004;137(6):1159-1160; author reply 1160-1161.

16. Hotta K, Hirakata A, Ohi Y, et al. Ultrasound biomicroscopy for examination of the sclerotomy site in eyes with proliferative diabetic retinopathy after vitrectomy. Retina. 2000;20(1):52-58.

17. Chalam KV, Shah GY, Agarwal S, Gupta SK. Illuminated curved 25-gauge vitrectomy probe for removal of subsclerotomy vitreous in vitreoretinal surgery. Indian J Ophthalmol. 2008;56(4):331-334.

18. Benitez-Herreros J, Lopez-Guajardo L, Camara-Gonzalez C, SilvaMato A. Influence of the interposition of a non-hollow probe during cannula extraction on sclerotomy vitreous incarceration in sutureless vitrectomy. Invest Ophthalmol Vis Sci. 2012;54(11):7322-7326.

19. Lopez-Guajardo L, Benitez-Herreros J, Camara-Gonzalez C, SilvaMato A. Assessment of vitreous incarceration in sclerotomies with optical coherence tomography, ultrasound biomicroscopy and direct visualization. Ophthalmic Surg Lasers Imaging. 2012;43(12):S117-S122.

20. Olsen TW, Sanderson S, Feng X, Hubbard WC. Porcine sclera: thickness and surface area. Invest Ophthalmol Vis Sci. 2002;43(8):2529-2532.

21. Olsen TW, Aaberg SY, Geroski DH, Edelhauser HF. Human sclera: thickness and surface area. Am J Ophthalmol. 1998;125(2):237-241.

22. Nicoli S, Ferrari G, Quarta M, et al. Porcine sclera as a model of human sclera for in vitro transport experiments: histology, SEM, and comparative permeability. Mol Vis. 2009;15(2):259-266.

23. Lee B, Litt M, Buchsbaum G. Rheology of the vitreous body: part 3. Concentration of electrolytes, collagen and hyaluronic acid. Biorheology. 1994;31(4):339-351.

24. Mihailovic Z, Atanasijevic T, Popovic V, Milosevic MB. Could lactates in vitreous humour be used to estimate the time since death? Med Sci Law. 2011;51(3):156-160.

25. López-Guajardo L, Pareja-Esteban J, Teus-Guezala MA. Oblique sclerotomy technique for prevention of incompetent wound closure in transconjunctival 25-gauge vitrectomy. Am J Ophthalmol. 2006;141(6): 1154-1156.

26. Bhende M, Agraharam SG, Gopal L, et al. Ultrasound biomicroscopy of sclerotomy sites after pars plana vitrectomy for diabetic vitreous hemorrhage. Ophthalmology. 2000;107(9):1729-1736.

27. Yeh PT, Yang CM, Yang CH, Huang JS. Cryotherapy of the anterior retina and sclerotomy sites in diabetic vitrectomy to prevent recurrent vitreous hemorrhage: an ultrasound biomicroscopy study. Ophthalmology. 2005;112(12):2095-2102.

28. Benitez-Herreros J, Lopez-Guajardo L, Camara-Gonzalez C, SilvaMato A. Influence of the sclerotomy use on mechanical incision competency in experimental model of vitrectomized eyes. Curr Eye Res. 2012;37(2):120-123. 


\section{Publish your work in this journal}

Clinical Ophthalmology is an international, peer-reviewed journal covering all subspecialties within ophthalmology. Key topics include: Optometry; Visual science; Pharmacology and drug therapy in eye diseases; Basic Sciences; Primary and Secondary eye care; Patient Safety and Quality of Care Improvements. This journal is indexed on
PubMed Central and CAS, and is the official journal of The Society of Clinical Ophthalmology (SCO). The manuscript management system is completely online and includes a very quick and fair peer-review system, which is all easy to use. Visit http://www.dovepress.com/ testimonials.php to read real quotes from published authors. 